Upcoming: November 29-December 3, MRS Fall Meeting, MRS.

To list an event in the Calendar, contact J. Meiksin, Materials Research Society, 506 Keystone Drive, Warrendale, PA 150867573; 724-779-3004 ext. 522; $\operatorname{tax} 724-779-8313$.

MRS, A-MRS, C-MRS, E-MRS, MRS-A, MRS-I, MRS-J, MRS$K$, MRS-R, MRS-T, M-MRS, or IUMRS at the end of an entry indicates sponsorship or co-sponsorship of an event by the Intemational Union of Materials Research Societies or one of its adhering bodies. "Endorsed" identifies events endorsed by MRS.

$\nabla$ identilies a new or revised entry this month.

\section{See the May 1999 MRS BULLETIN for July 1999 Calendar entries.}

\section{AUGUST 1999}

1-5 9th Intl. Conf. on Environmental Degradation of Materials in Nuclear Power Systems-Water Reactors, Newport Beach, CA. TMS Customer Service Dept., 184 Thorn Hill Rd., Warrendale, PA 15086; 724-776-9000; fax 724776-3770; e-mail csc@tms.org; http://www.tms.org/meetings/ meetings.htm].

1-5 Conf. on Microscopy and Microanalysis, Portland, $O R$. Microscopy Society of America Meeting Mgmt., c/o Rebedeau Group, 7000 W. Southwest Hwy., Chicago Ridge, IL 60415; 708361-6045; fax 708-361-6166; $\theta$-mail MSAMeetingManager@ MSA.microscopy.com; http://www. msa.microscopy.com.

1-6 11th Conf. on Crystal Growth and Epitaxy, Tucson, AZ. T. Gentile, ACCGE-11 Secretariat, American Assoc. for Crystal Growth, P.O. Box 3233, Thousand Oaks, CA 91359-0233; 805-492 7047: fax 805-492-4062; e-mail aacg@lafn.org; http://www.aml. arizona.edu/aacg.

2-6 $\nabla$ 18th Congress of the Intl. Commission for Optics: Optics for the Next Millennium, San Francisco, CA. SPIE, P.O. Box 10 , Bellingham, WA 98227-0010; 360676-3290; $\operatorname{tax} 360-647-1445$; e-mail cio18@spie.org http://www.spie.org.
2-6 $\nabla$ Conf. on Investigations and Applications of Severe Plastic Deformation, Moscow, Russia. T. Lowe, MST-D0, Los Alamos Nati. Lab., Los Alamos, NM 87545; 505-665-1131; 505-665-6584; e-mail tlowe@ianl.gov; http://nato. lanl.gov.

2-6 Denver $X$-ray Conf. Steamboat Springs, $C O$ D. Flaherty, Conf. Coordinator, ICDD, 12 Campus Blvd. Newtown Square, PA 19073-3273. 610-325-9814; fax 610-325-9823; e-mail flaherty@icdd.com http://www.dxcicdd.com.

4-13 18th Intl. Union of Crystallography Congress, Glasgow, Scotland, UK. Northern Networking Ltd. Congress Centra Office, Bellway House, 813 South Street, Glasgow G14 OBX, Scotland, UK; 44-141-954-4441: fax 44-141-954-2656; e-mail crystal@glasconf.demon.co.uk; http://www.chem.gla.ac.uk/ucr 99 .

6-10 $\nabla$ 8th Intl. Conf. on lon Sources, Kyoto, Japan. J. Ishikawa; e-mail icis99@mvtpc3. kuee.kyoto-u-ac.jp.

8-13 2nd Intl. Alloy Cont. Davos, Switzerland. Engineering Foundation Conferences, Three Park Ave., 27th Floor, New York, NY 10016; 212-591-7836; fax 212 591-7441; e-mail engfnd@aol com http://www.uefoundation.org.

9-11 Intl. Workshop on Dense Gas Dynamics, Boulder, CO. Inst. for Advanced Physics, 10875 U.S. Hwy. 285, Ste. 199, Conifer, CO 80433; e-mail dgd99@|APhysics. org; http://www.lAPhysics.org/ conferences/dgd99/.

\section{9-13 $\nabla$ 9th Canadian}

Semiconductor Technology Conf., Ottawa, Ontario, Canada. Sean McAlister Natl. Research Council of Canada, Ottawa, Ontario, Canada; 613-993-2514; fax 613-990-0202; e-mail sean. mcalister@nrc.ca.

9-13 12th Intl. Conf. on Textures of Materials, Montreal, Quebec, Canada. J.A. Szpunar, ICOTOM 12, Dept. of Metallurgica Engineering, McGill Univ. 3610 University St., Montreal, $\mathrm{PO}$ Canada, H3A 2B2.

15-20 2nd Conf. on Thermal Spray Processing of Nanoscale Materials, Quebec City, Canada. United Engineering Foundation, Three Park Ave., New York, NY 10016-5902; 212-591-7836; fax 212-591-7441; e-mail engfnd@aol.com; http://www. engfund.org.
19-20 $\nabla$ Conf. on Hot Workability of Aluminum Alloys, Composites, and Magnesium Alloys, Montreal, Quebec, Canada. H.J. McQueen, Matls. Man., Concordia Univ., Mechnical Engineering Hall, Bldg. $\mathrm{H}-549$ 1455 de Maisonneuve Blvd W. Montreal, Quebec H3G 1M8 Canada; 514-848-3145; fax 514848-3175; e-mail mcqueen@vaxw. concordia.ca; http://www.me. concordia.ca.

22-26 Fall Nati. ACS Meeting, New Orleans, LA. Div. of Meetings, American Chemical Society, 1155 16th St. N.W., Washington, DC 20036; 202-872-4396; fax 202872-6128; e-mail natlmtos@acs. org; http:/hww.acs.org

22-27 18th Intl. Conf. on Amorphous and Microcrystalline Semiconductors, Snowbird, UT. ICAMS18, Univ. of Utah, Dept. of Physics, 115 S. 1499 E. Rm. 201 Salt Lake City, UT 84112-0830; 801-581-4246; fax 801-581-4801; ICAMS18@mail.physics.utah.edu http://icams18.physics.utah.edu.

23-25 $\nabla$ NATO Workshap on Defects and Surface-Induced Effects in Advanced Perovskites, Riga, Latvia. E. Kotomin; e-mail ekotomin@latnet.Iv; http://www. cfi.lu.lv/nato.

23-27 10th Inti. Conf. on Rapidly Quenched and Metastable Materials, Bangalore, India. S.

Ranganathan and K. Chattopadhyay Dept. of Metallurgy, Indian Inst. of Science, Bangalore 560012, India; 91-80-309-2678; fax 91-80-331-

2991; e-mail raten@metalrg.ïsc ernet.in; http:// www. metalrg.iisc ernet.in/raten.

23-27 18th Intl. Conf. on $X$-Ray and Inner-Shell Processes Chicago, IL. X-99 Conf. Office,

Physics Div., Argonne Natl. Lab. Bldg. 203, Rm. G-122, $9700 \mathrm{~S}$. Cass Ave., Argonne, IL 604394843; 630-252-4044; fax 630-252 2864; e-mail x99@anl.gov http://www.phy.anl.gov/x99

23-27 $\nabla$ Intl. Cont. on Luminescence and Optical Spectroscopy of Condensed Matter, Osaka, Japan. T. Kushida Graduate School of Materials Science, Nara Inst. of Science and Technology, Ikoma, Nara 6300101, Japan; 81-743-72-6010 fax 81-743-72-6011; e-mail ICL99off@ms.aist-nara.ac.jp.

25-28 $\nabla$ 2nd Intl. Conf. on Laser Physics and Quantum Optics, Shanghai, China. S.Y. Zhu, Hong Kong Baptist Univ., Dept. of Physics, Hong Kong; 852-23397029; fax 852-2304-6558; e-mail syzhu@net2.hkbu.edu.hk
27-29 1st intl. Conf. on Scanning Probe Microscopy of Polymers, Santa Barbara, CA. T. Mehr, Conf. Coordinator, 112 Robin Hill Rd., Santa Barbara, CA 93117; 805-967-1401; e-mail terry@di.com; www.di.com/poly/ poly.html.

$29-3 \nabla 7$ th Intl. Conf. on Ferroelectric Liquid Crystals, Darmstadt, Germany. FLC 99, C. Jachem, Condensed Matter Research Group, Inst. of Physical Chemistry, Darmstadt Univ. of Technology, Petersenstrasse 20, D-64287 Darmstadt, Germany; 49 $61-51-164945 ; 49-61-51-164924$ e-mail flc99@tu-darmstadt.de.

30-1 $\nabla$ Conf. on Coatings for Asia, Singapore. SSPC, 40 24th St, Sixth Floor, Pittsburgh PA 15222; 412-281-2331; fax 412 281-9993; http://www.sspc.org.

30-2 $\nabla$ European Corrosion Congress, Aachen, Germany. K. Müller, DECHEMA e.V. TheodorHeuss-Allee 25, D-60486, Frankfurt am Main, Germany; 4969-7564-242; fax 49-69-7564-304 e-mail tagungen@dechema.de.

30-3 11th Intl. Conf. on Thin Films, Cancun Mexico. ICTF11/SMCSVC-19, Dept. de Fisica Centro de Investigacion y de Estudios Avanzados del I.P.N.P.O Box 14-740, Mexico 07000 D.F. Mexico; 52-5-7477000; fax 52-5-7477096; e-mail ictf99@fis. cinvestav.mx

30-3 Conf. on Lasers and Electro-Optics/Pacific Rim, Seoul, Korea. Optical Society of Korea Rm. 811, Korea Science \& Technology Ctr., 635-4, Yucksamdong, Kangnam-ku, Seoul 135 703, Korea; 82-2-3452-6560 fax 82-2-3452-6561.

\section{SEPTEMBER 1999}

1-3 OSA Topical Meeting on Nonlinear Guided Waves and Their Applications, Dijon, France. Optical Society of America, Conf. Services Dept., 2010 Massachusetts Ave. N.W. Washington, DC 20036 1023; 202-416-1980; fax 202-4166100; e-mail confserv@osa.org; http://www.osa.org/mtg-conf.

1-4 Surfaces in Biomaterials Conf., Scottsdale, $A Z$. Surfaces in Biomaterials Foundation, 13355 10th Ave. N., Minneapolis, MN 55441; 612-915-1011; fax 612-927-8127; http://www surfaces.org.

2-4 15th Annual Meeting of the Academy of Surgical Research, Scottsdale, $A Z$. The Academy of Surgical Research, 13355 10th Ave. North, Ste. 108, Minneapolis, MN 55441-5510; 612-545-1919; fax 612-545-0335; http://www. surgicalresearch.org.

2-8 6th Intl. Conf. on Hydrogen Materials Science and Chemistry of Metal Hydrides, Katsiveli Yalta, Ukraine. D.V. Schur, S. Yu Zaginaichenko Program Committee ICHMS'99, P.0. Box 195, Kiev-150, 252150 , Ukraine; phone/fax 380-44-4440001; e-mail shurzag@ ipms.kiev.ua.

\title{
5-7 ICSSS-International
}


6-9 Euro-Fillers ' 99 Conf. Lyon-Villeurbanne, France. Euro-Fillers ' 99 Secretariat, Lab. des Matériaux, Macromoléculaires, Inst. Natt. des Sciences Appliquées, Bát. 403-20, Ave. Albert Einstein, 69621 Villeurbanne Cedex, France; 33-4-7243-8979; tax 33-4-7243-8527; e-mail eurofillers@insa-lyon.fr.

6-10 8th intl. Conf. on Ion Sources, Kyoto, Japan. J. Ishikawa, Electronic Science and Engineering Dept., Kyoto Univ. Yoshida-hommachi, Sakyo-ku, Kyoto 606-8501, Japan; 81-75753-5325; fax 81-75-753-5324; e-mail icis99@mutpc3.kuee kyoto-u ac; http://mvtpc3.kuee. kyoto-u.ac.jp/icis99.

0-10 $\nabla$ SEMI/IEEE Advanced Semiconductor Manufacturing Conf., Boston, MA. SEMI North America, $805 \mathrm{E}$. Middlefield Rd. Mountain View, CA 94043-4080 650-964-5111; tax 650-967-5375; e-mail semihq@semiorg;

http://www.semi.org.

8-10 $\nabla$ Surface Modification Technologies Conf., and Processing and Fabrication of Advanced Materials Conf. Singapore. SMT XIII/PFAM VIII Conf Secretariat Conf Mgmt Centre, Administration Annex \#04-06, Nanyang Ave., Singapor 639798, Republic of Singapore; 65-790-4723; fax 65-793-0997; e-mail SMTPFAM99@ntu.edu.sg.

10-15 10th Intl. Conf. on Recent Progress in Many-Body Theories Seattle, WA. A. Bulgac, Dept. of Physics, Univ. of Washington, P.O. Box 351560 , Seattle, WA 98195 1560; fax 206-685-9829; e-mail mbx@phy. washington.edu: http:/www. phys.washington. edu/ mbx.

12-16 4th Intl. Cont on Nuclear Physics at Storage Rings, Bloomington, IN. J. Meadows, Indiana Univ. Cyclotron Facility, 2401 Milo B. Sampson Lane, Bloomington, IN 47408; 812-8559365; 812-855-6645; e-mail stori99@iuct.indiana.edu.

12-17 $\nabla$ 6th Intl. Conf. on Surface $X$-ray and Neutron Scattering, Noordwijkerhout, The Netherlands. 6SXNS Secretariat, Philips Research Labs WB33, Prot Holstlaan 4, 5656 AA Eindhoven, The Netherlands; 31-40-2744551; fax 31-40-2745002; e-mail deboerd@natlab.research. philips.com.
12-17 $\nabla$ Intl. Joint Conf. on Silicon Epitaxy and Heterostructures: 8th Intl. Symposium on Silicon Molecular Beam Epitaxy, and 3rd Intl. Symposium on Silicon Heterostructures, Zao, Miyagi, Japan. J. Murota, Steering Committee Chairman, IJC-Si, Research Inst. of Electrical Communication, Tohoku Univ. 2-1-1 Katahira, Aoba-ku, Senda 980-8577, Japan; 81-22-2175548; $\operatorname{tax} 81-22-217-5565$ e-mail IJC-Si@murota.riec tohoku.ac.jp; http://IJC-Si.murota riec.tohoku.ac.jp.

13-15 4th Intl. Conf. on Spray Forming, Baltimore, MD. J.

Abrahams, Conf. Secretariat, "Beggars Roost," Channels End Rd., Colmworth, Bedfordshire MK44 2NS, UK; 44-12-34-37-8862 tax 44-12-34-37-6219; $\theta-\mathrm{ma}$ 100700.2023@compuserve.com.

13-17 7th Intl. Conf. on Microwave and High Frequency Heating, Valencia, Spain. 7th Intt. Conf. Microwave \& HF Heating, TOT NOU, C/Cirilo Amorós, 27-2 ${ }^{\circ}$-C E-46004 Valencia, Spain; 34-96394-3445; fax 34-96-394-1654 e-mail microwavetsitupves: http://www.digital.es/icom/totnou

14-15 \ SEMI Chip Scale Intl. Conf., San Jose, CA. J. Sullivan, SEMI North America, $805 \mathrm{E}$. Middlefield Rd., Mountain View, CA 94043-4080; 650-964-5111. tax 650-967-5375; e-mail jsullivan. semiorg; http://www.semi.org.

15-17 $\nabla$ SEMICON/Taiwan 99, Taipei, Taiwan. SEMI North America, $805 \mathrm{E}$. Middlefield Rd. Mountain View, CA 94043-4080 650-964-5111; fax 650-967-5375; e-mail semihq@semiorg;

http://www.semi.org.

19-23 Specialty Conf. on Copper, Phoenix, AZ. Custome Service Dept. TMS 184 Thorn Hill Rd., Warrendale, PA 15086; 724776-9000; fax 724-776-3770; e-mail csc@tms.org; http://www. tms.org.

\section{0-24 3rd Conf. of the} Yugoslav Materials Research Society, Herceg Novi, Yugoslavia. Yu-MRS Conf. Secretariat, Inst. of Technical Sciences of the Serbian Academy of Sciences and Arts. Knez Mihailova 35/IV, P.O. Box 745,11000 Belgrade, Yugoslavia 381-11-185-437; fax 381-11-185263; e-mail yumrs@itn.sanu.ac.yu; http://www.yu-mrs.org.yu

20-24 $>7$ th Intl. Conf. an Quasicrystals, Stuttgart, Germany. ICQ7'99 Conf. Secretariat, Inst. für Theoretische und Angewandte

Physik, D-70550 Stuttgart

Germany; 49-711-685-5253; fax 49-711-685-5271; e-mail

icq7@itap.physik.uni-stuttgart.de;

http://icq7.uni-stuttgart.de.
20-25 $\nabla$ 3rd Intl. Symposium on Vacuum Technology and Equipment, in conjunction with 4t Intl. Symposium on Diamond and Related Films, Kharkov, Ukraine. E.I. Savtchenko, P.O. Box 10363 , Kharkov 310108, Ukraine: fax 380-572-35-35-29; e-mail vacuum_org@kipt.kharkov.ua; www.kipt.kharkov.ua.

\section{1-23 1st Intt. SAMPE}

Automotive Conf., Meeting of the Engineering Society, and the Engineering Society/Society of Automotive Engineers Intl. Body Engineering Conf, Detroit, MI C.L. Hammermesh, Technical Director Society for Advancement of

Material and Process Engineering. SAMPE Intl. Business Office, P.O. Box 2459, Covina, CA 91722-8459; 626-331-0616; fax 626-332-8929.

21-23 $\nabla$ Coating Conf., Dallas, $T X$. V. Thatcher, Conf. Coordinator P. O. Box 54464, Cincinnati, OH 45254; 513-624-9988; fax 513624-0601; www.finishing. com/coating.

21-24 18th European Conf. on Surface Science, Vienna, Austria. ECOSS-18 Secretary Inst fur Allgemeine Physik, TU-Wien Wiedner Hauptstr. 8-10/E134, A-1040 Vienna, Austria.

22-24 SPIE Intl. Symposium on Microelectronic Manufacturing. Santa Clara, CA. SPIE P. O. Box 10. Bellingham, WA 98227-0010; 360-676-3290; $\operatorname{tax} 360-647-1445$ e-mail spie@spie.org; $h t t p / / w w w$. spie.org/info/mm.

$23 \nabla$ Meeting of ASTM Committee E-37 on Thermal Measurements, Savannah, GA. T. O'Toole, ASTM, 100 Barr Harbor Dr. West Conshohocken, PA 19428-2959; 610-832-9739. fax 610-832-9635; e-mail totoole@astm.org; www.astm.org.

24-26 OSAACS Topica Meeting on Organic Thin Films for Photonics Applications; with OSA Topical Meeting on Bragg Coatings, Photosensitivity, and Poling in Glass Waveguides:

Santa Clara, CA Optical Society of America, Conf. Services Dept. 2010 Massachusetts Ave., N.W. Washington, DC 20036-1023;

202-416-1980; fax 202-416-6100; e-mail confserv@osa.org; http://www.osa.org/mtg-cont

26-29 $\nabla$ Intl. Seminar on Technology of Inherently Conductive Polymers, Toronto Ontario Canada M. Aldissi, 813854-4332; tax 813-854-5596; e-mail fractals@infobridge.com; whw fastwrw com/conductive polymers.
26-1 OSA Annual Meeting, and Interdisciplinary Laser Science Conf., Santa Clara, CA. Optical Society of America, Cont. Services Dept., 2010 Massachusetts Ave. N.W. Washington, DC 20036 1023; 202-416-1980; tax 202-416 6100; e-mail contsen@osa.org http://www.osa.org/mtg-cont.

27-29 1st Intl. SAMPE

Automotive Conf., with ESD Advanced Coatings Technology Conf Detroit, MI Society for the Advancement of Material and Process Engineering, 1161 Parkview Dr., Covina, CA 91724 3748 ; 626-331-0616; fax 626-332 8929; e-mail sampeibo@aol.com; muw.et.byu.edu/ sampe.

27-29 $\nabla$ 4th Intl. Cont. on Cutting Tools: Market Trends, Forecasts, and Advances in Technology, Chicago, IL. C. Jones Gorham Advanced Materials Inst., P. O. Box 250, Gorham, ME 04038 0250; 207-892-5445; tax 207-892 2210; e-mail gorham@goradv.com.

27-30 $\nabla 25$ th European Conf. on Optical Communications, Nice France. G. Marsot, Société des électriciens et des électroniciens, 48, rue de la Procession, F-75724 Paris Cedex 15, France; 33-1 4449-6000; fax 33-1-4449-6049.

27-30 European Cont. on Advanced Materials and Processes, Munich, Germany. EUROMAT Congress Office, c/o Deutsche Gesellschaft für Materialkunde, Hamburger Allee 26, D-60486 Frankfurt, Germany; 49-69-7917-750; fax 49-69-7917733; http //Www.fems.org.

27-1 $\nabla$ 8th Intl. Topical Meeting on Optics of Liquid Crystals Humacao, Puerto Rico. F. Aliev, Resource Center for Science and Engineering, P.0. Box 233343334, San Juan, Puerto Rico 00931; 757-765-5170; fax 787756-7717; e-mail olc99@adam. uprr.pr; http://web.uprr.pr/olc99.

27-1 $\nabla$ 12th Intl. Cont. on Ternary and Multinary Compounds, Hsin-Chu, Taiwan. B.H. Tseng inst of Materials Science, Natl. Sun Yat-Sen Univ., Kaohsiung 807, Taiwan; 886-7525-4056; fax 886-7-5254099; e-mail baeheng@mail.nsysu. edu.w; http:/huww.mse.nsysu. edu.tw/ictmc12.

28-30 Advanced Metallization Cont., Orlando, FL. Univ. of Calif. Berkeley Extension, 1995

University Ave., Berkeley, CA 94720-7010; 510-642-4151; fax 510-642-6027; e-mail course@ unx berkeley.edu.

\section{OCTOBER 1999}

4-7 IEEE Intl. Silicon-onInsulator Conf., Rohnert Park, CA. BACM, 520 Washington Blvd., Ste. 350, Marina del Rey, CA 90292. 310-305-7885; tax 310-305-1038; e-mail bacm@mediaone.net; 
17-19 $\nabla$ Conf. on Fine, Ultrafine, and Nano Powders, Orlando, FL. M.N. Rittner, Business Communications $\mathrm{Co}_{0 .}$ Inc.; 847 298-3754; fax 847-298-0524 e-mail mrittner@megsinet.net.

17-22 196th Meeting of The Electrochemical Society and Fal Meeting of The Electrochemical Society of Japan, Honolulu, HI. The Electrochemical Society. 10 S. Main St., Pennington, NJ 08534-2896; 609-737-1902; fax 609-737-2743; e-mail ecs@ electrochem.org; http://www. electrochem.org.

19-20 SEMICON/Southwest, Austin, $T X$. J. Sullivan, SEMI North America, $805 \mathrm{E}$. Middlefield Rd. Mountain View, CA 94043-4080 650-964-5111; fax 650-967-5375; e-mail jsullivan@semiorg; http://www.semi.org.

19-21 ₹ Intl. Conf. for Students, Young Specialists, and EngineersOptics 99, St. Petersburg, Russia. V.M. Arpishkin, D.S. Rozhdestvensky Optical Society, 12 Birzhevaya line, St. Petersburg 199034, Russia; phone/tax 7-812218-1335; e-mail arpish@ros.spb.ru.

21-22 $\nabla$ Conf. on Microscale Characterization for Materials Discovery, Newark, DE. Engineering Outreach, College of Engineering, Univ. of Delaware, Newark, DE 19716-3101: 302-831-4863; fax 302-831-8179; e-mail outreach@ mvs.udel.edu; http//www.udel.edu/ engg/DeptsPrgms/EO/.

22-23 $\nabla$ 1st Intl. Workshop on Dielectric Thin Films for Future ULSI Devices: Science and Technology, Tokyo, Japan. K. Shiraishi; fax 81-462-40-4317; e-mail siraisi@will.brl.ntt.co.jp; http://www.ipc. hiroshima-u. ac.jp/_semicon/IWDTF/.

22-23 $\nabla$ 8th Topical Conf. on Quantitative Surface Analysis, Puyallup, WA. C. Powell, Chemistry B-364, NIST

Gaithersburg, MD 20899; 301 975-2534; fax 301-926-6689 Q-mail cedric.powell@nist.gov.

25-27 2nd Intl. Symposium on Adhesion Measurement of Thin Films and Coatings, Newark, NJ. R.H. Lacombe, Conf. Chairman, 3 Hammer Dr., Hopewell Junction NY 12533; 914-227-7026; fax 212656-1016; $\theta$-mail thlacombee compuserve.com; http://mstconf. com/adhmeas.htm.

25-29 $\nabla$ 3rd Intl. Symposium on Control of Semiconductor Interfaces, Karuizawa, Japan. T. Katoda, Conf. Secretary

Dept. of Electronic and Photonic System Eng., Kochi Univ. Tech. Tosayamada, Kochi 782-0003 Japan; 81-8875-3-1010; fax 818875-7-2120; e-mail katouda@ ele.kochi-tech.ac.jp.
25-29 AVS 46th Intl. Symposium, Boston, MA American Vacuum Society, 120 Wall St., 32nd Floor, New York, NY 10005-3993; 212-248-0200; fax 212-248-0245; e-mail avsnyce vacuum.org; http//wuw.vacuum.org

26-28 32nd Intl. Symposium on Microelectronics, Chicago, IL. Intl. Microelectronics and Packaging

Society, 1850 Centennial Park Dr Ste. 105, Reston, VA 20191-1517; 703-758-1060; fax 703-758-1066; e-mail imaps@imaps.org; www.imaps.org.

26-30 31st Intl. SAMPE

Technical Conf., with the

Composite Fabricators

Association, Chicago, IL. Society for the Advancement of Material and Process Engineering, 1161 Parkview Dr., Covina, CA 91724 3748; 626-331-0616; fax 626-332 8929; e-mail sampeibo@aol.com; www.et.byu.edu/ sampe.

27-31 $\nabla$ 31st Intl. SAMPE Technical Conf. on Advanced Materials and Processes, Chicago, IL SAMPE Intl. Business Office P.0. Box 2459, Covina, CA $91722-$ 8459; 800-562-7360; fax 626-3328929; e-mail sampeibo@aol.com; http://www.byu.edu/ sampe.

28-29 Intl. Symposium on Adhesion Aspects of Thin Films, Newark, NJ. R.H. Lacombe, Conf. Chairman, 3 Hammer Dr. Hopewell Junction, NY 12533; 914-227-7026; tax 212-656-1016; e-mail rhlacombe@compuserve. com; http://mstconf.com/adhfilm. htm.

31-3 14th Annual National Educators' Workshop, Auburn Hilts, MI. J. Jacobs, School of Jechnology, Norfolk State Univ. 2401 Cornrew Ave. Nortolk. VA 23504 ; 757-683-8109; fax 757-6838215; e-mail j_jacobs@vger.nsu. edu; http://coulomb.nsu.edu/new.

31-4 $\nabla$ TMS Fall Meeting Cincinnati, OH. TMS, 184 Thorn Hill Rd. Warrendale, PA 15086: 724-776-9000; fax 724-776-3770; e-mail csc@tms.org; http://www. tms.org.

\section{NOVEMBER 1999}

1-3 3ro North American Conf. on the Science and Technology of Emulsion Polymers/Polymer Colloids, Hilton Head Island, SC. Inst. of Materials Science, 75 S. Manheim Blvd., New Paltz, NY 12561; 914-255-0757 fax 914-255-0978; e-mail nainquiry@ims-np.org/narcCol.
1-3 Intl. Conf. on the Science, Technology, and Applications of Sintering, State College, $P A$ R. Cornwall, Materials Research Lab. 277 MRL, Pennsylvania State Univ., University Park, PA 16802 . 814-863-8735; fax 814-863-9704; e-mail rgc5@psu.edu; http:/www. pmlab.psu edu/sint99.

\section{1-4 ASM Intl. Materials} Solutions Conf., Cincinnati, $\mathrm{OH}$ ASM Intl., Materials Park, $\mathrm{OH}$ 44703-0002; 440-338-5151; fax 440-338-4634; www.asm-intl.org.

\section{3-5 $\nabla$ Chemical Process} Development Workshop, Frazer, $P A$. Cauldron Process Chemistry 383 Phoenixville Pike, Malvern, PA 19355; 610-727-2215; tax 610 727-5762.

8-10 3rd ISOPE Ocean Mining Symposium, Goa, India. Intl. Society of Offshore and Polar Engineers, P.0. Box 1107, Golden CO 80402-1107; 303-420-8114: fax 303-420-3760.

8-10 $\nabla$ North American Research Conf. Series: Polymer Stabilizers and Modifiers, Hilton Head Island, SC. Inst. of Materials Science, P.O. Box 369 , New Paltz, NY 12561; 914-255-0757; fax 914-255-0978; e-mail nainquiry@ims-np.org.

http:/thww.ims-np.org/

15-18 2nd Intl. Conf. on Processing Materials for

Properties, San Francisco, CA Customer Service Dept., TMS, 184 Thorn Hill Rd. Warrendale, PA 15086; 724-776-9000; $\operatorname{tax} 724$ 776-3770; e-mail csc@tms.org; http://www.tms.org

15-18 18th Intl. Congress on Applications of Lasers and ElectroOptics, San Diego, CA. Laser Inst. of America, 12424 Research Pkwy. Ste. 125, Orlando, FL 32826; 407-380-1553; fax 407-380-5588; e-mail lia@Laserlnstitute.org www.LaserInstitute.org

15-18 $\nabla$ 44th Annual Conf. on Magnetism and Magnetic Materials, San Jose, CA. MMM Conf. Coordinators, Courtesy Associates, 2000 L St. NW, Ste. 710, Washington, DC 20036; 202973-8668; fax 202-973-8722; e-mail magnetism@courtesyassoc. com; http://www.magnetism.org.

29-1 Intl. Symposium on Polyimides and Other High Temperature Polymers: Synthesis, Characterization, and Applications, Newark, NJ. R.H. Lacombe, Conf. Chairman 3 Hammer Dr.

Hopewell Junction, NY 12533 914-227-7026; fax 212-656-1016; e-mail mlacombe@compuserve. com; http://mstconf.com/ polyimd.htm.
29-3 MRS Fall Meeting, Boston, MA. Materials Research Society, 506 Keystone Drive, Warrendale, PA 15086-7573; 724-779-3003; fax 724-779-8313. http:/www.mrs.org/. MRS

\section{DECEMBER 1999}

$1-3 \nabla$ 2nd Asia/Australia Regional Meeting of Polymer Processing Society, Bangkok, Thailand. http:/www.ops.mtec. or.th.

1-3 SEMICON Japan 99 Chiba, Japan. J. Sullivan, SEM North America, 805 E. Middlefield Rd. Mountain View, CA 940434080; 650-964-5111; fax 650-9675375; e-mail jsullivan@semiorg: http://www.semi.org.

2-3 7th Intl. Symposium on Metallized Plastics: Fundamental and Applied Aspects, Newark, NJ. R.H. Lacombe, Cont. Chairman, 3 Hammer Dr., Hopewell Junction NY 12533; 914-227-7026; fax 212 656-1016; e-mail thlacombe@ compuserve.com; http://mstconf $\mathrm{com} /$ metalpla.htm.

6-8 IEEE Intl. Electron Devices Meeting, Washington, DC. http://ieee.oprg/conterence/iedm.

6-8 Intl. Conf. on Computational Fluid Dynamics in Minerals and Process Industries, Melboume Australia. P. Schwar, CSIRO Minerals, Box 312, Clayton South, Victoria 3169. Australia; 613-9545 8500; fax 613-9562-8919; e-mail cfd@minerals.csiro.au; http://mww. minerals.csiro.au.

12-15 $\nabla$ 2nd Intl. Workshop on Mechanics, Physics, and Reliability of Polymeric Materials for Microelectronic and Photonic Applications, Paris, France. B. Bigalke, Meetings and Confs. ASME, 3 Park Ave., New York, NY 10016-5990; 212-705-7057; fax 212-705-7856; e-mail bigalkeb@ asme.org.

\section{FEBRUARY 200}

7-9 2000 IEEE Intl. Solid-State Circuits Conf., San Francisco, CA D. Suiters; 202-331-2000; e-mail isscc@courtesyassoc.com

11-14 OSA Vision Science and Its Applications Topical Meeting. collocated with OSA Laser Applications to Chemical and Environmental Analysis Topical Meeting, Santa Fe, NM. Optical Society of America, Conf. Services Dept., 2010 Massachusetts Ave., N.W., Washington, DC 200361023; 202-416-1980; fax 202-416 6100; e-mail confserv@osa.org http://www.osa.org/mtg-conf

14-16 $\nabla$ Microscopy and Microanalysis of Engineering Materials (MMEM2000), Melboume, Victoria, Australia. Astrid Nordmann, MMEM Conference Secretariat, Centre for Advanced Materials Technology, 
6-8 Polymer Stabilizers and Modifiers Cont., Hilton Head Island, SC. Inst. of Materials Science, $75 \mathrm{~S}$. Manheim Blvd. New Paltz, NY 12561; 914-2550757; tax 914-255-0978; e-mail nainquiry@ims-np.org/narcStab.

7-12 $>$ Optical Fiber Communication Conf., Baltimore MD. Optical Society of America, Conf. Services Dept., 2010 Massachusetts Ave. N.W. Washington, DC 20036-1023; 202-416-1980; fax 202-416-6100; e-mail confserv@osa.oro http://www.osa.org/mtg-conf.

12-16 $\nabla 129$ th TMS Annual Meeting, Nashville, TN. TMS, 184 Thorn Hill Rd., Warrendale, PA 15086; 724-776-9000; fax 724776-3770; e-mail wilson@tms.org http://www.tms.org

15-16 $\nabla$ SEMICON/China Beijing, China. SEMI North America, $805 \mathrm{E}$. Middlefield Rd. Mountain View, CA 94043-4080 650-964-5111; fax 650-967-5375 e-mail semihq@semiorg; http://www.semi.org.

20-24 APS 2000 March Meeting, Minneapolis, MN. Meetings Dept, American Physical Society, One Physics Ellipse, College Park, MD 20740-3844; 301-209-3286; http://www.aps.org.

\section{APRIL 2000}

2-7 2nd World Congress on Microwave Processing, Orlando. FL. D.E. Clark, Organizing Committee Chair, Dept. of Materials Science and Engineering, 136 MAE, Univ. of Florida, P.0. Box 116400, Gainesville, FL 32611-6400; 352-392-3660; fax 352-846-2033. Endorsed.

3-4 Microscopy of Composite Materials V, Oxford, UK. Royal Microscopical Society, 37/38 St. Clements, Oxford 0X4 1AJ, UK 44-1865-248768; fax 44-1865791237; e-mail meetings@rms org.uk; http://www.rms.org.uk.

3-6 $\nabla$ 3rd IntI. Cont. on Electromagnetic Processing of Materials, Nagoya, Japan. JATIS Sogo-Kojimachi, No. 3 BIdg., 1-6 Kojimachi, Chiyoda-Ku, Tokyo 102-0083, Japan; 81-3-32394804: fax 81-3-3239-4714 e-mail jatisc@msn.com.

11-13 Intl. Microscopy Conf. London, UK. Royal Microscopical Society, $37 / 38$ St. Clements, Oxford OX4 1AJ, UK: 44-1865248768; fax 44-1865-791237; e-mail meetings@rms.org.uk; http://www.rms.org.uk.
16-19 6th Intt. Multidisciplinary Conf.: Complexity and Fractals in the Sciences, Singapore. http://www.kingston. ac.uk/fractal

24-28 MRS Spring Meating, San Francisco, CA. Materials Research Society, 506 Keystone Drive, Warrendale, PA 150867573; 724-779-3003; fax 724-779 8313; http://www.mrs.org/. MRS.

\section{MAY 2000}

7-12 Conf. on Lasers and Electro-0ptics, and Quantum Electronics and Laser Science Conf., San Francisco, CA. Optical Society of America, Conf. Services Dept., 2010 Massachusetts Ave. N.W., Washington, DC 200361023; 202-416-1980; fax 202-4166100; e-mail confserv@osa.org http://www.osa.org/mtg-conf.

14-19 $\nabla$ 197th Meeting of The Electrochemical Society, Toronto, Ontario, Canada. The Electrochemical Society, $10 \mathrm{~S}$. Main St. Pennington, NJ 08534 2896; 609-737-1902; fax 609-737. 2743; e-mail ecs@electrochem. org; http://www.electrochem.org meetings/.

15-20 6th World Biomaterials Congress, Kamuela (Big /sland) HI. Society for Biomaterials. 1335510 th Ave. N., Ste. 108, Minneapolis, MN 55441; 612-9278108; fax 612-927-8127; e-mail biomaterials.org; http://www. biomaterials.org.

22-24 $\nabla$ 6th Intl. Conf. on Computer Aided Assessment and Control: Damage and Fracture Mechanics, Montreal, Canada. S Walsh, Conf. Secretariat, Wessex inst. of Technology, Ashurst Lodge, Ashurst, Southampton S040 7AA, UK; 44-1703-293-223; tax 44-1703-292-853; e-mail slwalsh@wessex.ac.uk; http://www.wessex.ac.ukd

22-27 26th Congress on Chemical Engineering, Environmental Protection, and Biotechnology, Frankfurt am Main Germany. DECHEMA Deutsche Gesellschaft fur Chemisches Apparatewesen, Chemische Technik und Biotechnologie e.V. Th. Scheuring, Germany; 49-697564-230; fax 49-69-7564-298; http://www.dechema.de.

28-2 10th Intl. Offshore and Polar Engineering Conf., Seattle, WA. Intl. Society of Offshore and Polar Engineers, P.0. Box 1107 Golden, CO 80402-1107; 303-4208114; fax 303-420-3760.
28-2 $\nabla$ Intl. Cont. on Transport Processes in Inorganic Materials: Fundamentals to Devices, Venice, Italy. CIMTEC, P.0. Box 174 48018 Faenza, Italy; 39-54622461; fax 39-546-664138.

\section{JUNE 2000}

18-23 $\nabla$ OSA Diffractive Optics and Micro-Optics Topical Meeting, collocated with OSA Optical Fabrication and Testing Topical

Meeting, Quebec City, Canada. Optical Society of America, Conf. Services Dept., 2010

Massachusetts Ave., N.W. Washington, DC 20036-1023; 202-416-1980; fax 202-416-6100; e-mail confserv@osa.org; http://www.osa.org/mtg-conf.

26-2 $\nabla$ Seminar on Defect Structures Evolution in Condensed Matter, Barnaul, Russia. EDS 2000, General Physics Dept., Altai State Technical Univ., 46 Lenin St. Barnaul, Russia 656099; 0073852-368-0522; fax 007-3852 260-516; e-mail asba@ab.ru (mailto:asba@ab.ru); http://www. dcn-asu.ru/structure/cdo/ index.ru.html.

\section{JULY 2000}

10-12 $\nabla$ OSA Optical Amplifiers and Their Applications Topical

Meeting, Ouebec City, Canada.

Optical Society of America, Conf. Services Dept., 2010

Massachusetts Ave., N.W.

Washington, DC 20036-1023.

202-416-1980; fax 202-416-6100; e-mail confserv@osa.org;

http://www.osa.org/mtg-conf.

10-13 $\nabla$ The Plutonium

Futures-The Science Conf.

Santa Fe, NM. A. Liesse, Plutonium

Futures-The Science, Los Alamos Natt. Lab., Nuclear Materials

Technology Div., P.0. Box 1663;

M.S. E500, Los Alamos, NM 87545; 505-665-5981; fax 505667-7966; e-mail pucont2000@ lanl.gov; http//www.lanl.gov/ pu2000.htm

13-15 $>$ OSA Integrated Photonics Research Topical Meeting, Quebec City, Canada. Optical Society of America, Conf. Services Dept., 2010

Massachusetts Ave., N.W. Washington, DC 20036-1023; 202-416-1980; fax 202-416-6100; e-mail confservosa.org; http://www.osa.org/mtg-conf.

16-20 $\nabla$ 5th Intl. Cont. on

Structural and Functional Intermetalfics, Vancouver, British Columbia. TMS, 184 Thorn Hill Rd. Warrendale, PA 15086; 724-776-9000; fax 724-776-3770; e-mail csc@tms.org; http://www. tms.org.
17-21 5th Intl. Cont. on Diffusion in Materials, Paris, France DIMAT 2000 DECM/ SRMP, Bat. 520, CEASACLAY, 91191 Gif-sur-Yvette Cedex, France; e-mail dimat2000@cea.fr; www.dimat2000.cea.fr.

24-28 $\nabla$ 18th Intl. Liquid Crystal Cont., Sendai, Japan. T. Uchida Executive Chair ILCC 2000, Dept. of Electronics, Graduate School of Engineering, Tohoku Univ. 05 Aza-aoba, Aramaki, Aoba-ku, Sendai 980-8579, Japan; 81-22217-7117; $\operatorname{tax} 81-22-263-9404$ e-mail ilcc2000@ecei.tohoku.ac.jp; http://wwwsoc.nacsis.ac.jp/ics/ ILCC2000.

30-4 SPIE Annual Meeting, San Diego, CA. SPIE, P.0. Box 10 , Bellingham, WA 98227-0010; 360-676-3290; fax 360-647-1445 e-mail spie@spie.org; http://www. spie.org.

\section{AUGUST 2000}

6-11 $\nabla 7$ th International Symposium on Polymer Electrolytes (ISPE7), Noosa, Queensiand, Australia. Astrid Nordmann, ISPE7 Conference Secretariat, Centre for Advanced Materials Technology, Monash University, Wellington Road,

Clayton, Victoria, 3168, Australia: 61-3-9905-5791; fax 61-3-99044998; e-mail ispe7@eng.monash. edu.au; http:/www.chem.monash. edu.au/electrolytes/ispe7/.

13-16 $\nabla$ Nonlinear Optics Topical Meeting, Maui, HI. Optical Society of America, Conf. Services Dept., 2010 Massachusetts Ave.,

N.W. Washington, DC 200361023; 202-416-1980; fax 202-4166100; e-mail confserv@osa.org; http://www.osa.org/mtg-conf.

22-26 $\nabla$ ab initio (from Electronic Structure) Calculations of Complex Processes in Materials Schwaebisch Gmuend, Germany. e-mail psik-coord@daresbury. ac.uk.

\section{SEPTEMBER 2000}

3-8 Congress of Histochemistry and Cytochemistry. York, UK. Royal Microscopical Society, 37/38 St. Clements, Oxford OX4 1AJ, UK; 44-1865248768; fax 44-1865-791237; e-mail meetings@rms.org.uk; http://www.rms.org.uk.

10-15 Conf, on Lasers and Electro-Optics/Europe, and Intl. Quantum Electronics Cont., Nice, France. Optical Society of America, Conf. Services Dept, 2010 Massachusetts Ave., N.W Washington, DC 20036-1023; 202-416-1980; $\operatorname{tax} 202-416-6100$ e-mail confserv@osa.org; http://www.osa.org/mtg-cont

17-21 $\nabla$ th Intl. Symposium on Superalloys, Champion, PA. TMS, 184 Thorn Hill Rd. Warrendale, PA 15086; 724-776-9000; fax 724776-3770; e-mail csc@tms.org; http://www.tms.org

17-22 13th Intl. Cont. on lon Implantation Technology, Tyrol 